\section{Kicking Performance in Young U9 to U20 Soccer Players: Assessment of Velocity and Accuracy Simultaneously}

\author{
Luiz H. P. Vieira, Sérgio A. Cunha, Renato Moraes, Fabio A. Barbieri, Rodrigo
} Aquino, Lucas de P. Oliveira, Martina Navarro, Bruno L. S. Bedo \& Paulo R. P. Santiago

To cite this article: Luiz H. P. Vieira, Sérgio A. Cunha, Renato Moraes, Fabio A. Barbieri, Rodrigo Aquino, Lucas de P. Oliveira, Martina Navarro, Bruno L. S. Bedo \& Paulo R. P. Santiago (2018) Kicking Performance in Young U9 to U20 Soccer Players: Assessment of Velocity and Accuracy Simultaneously, Research Quarterly for Exercise and Sport, 89:2, 210-220, DOI: 10.1080/02701367.2018.1439569

To link to this article: https://doi.org/10.1080/02701367.2018.1439569

巴follished online: 07 Mar 2018.

Submit your article to this journal $\sqsubset$

Џll Article views: 289

View Crossmark data $₫$ 


\title{
Kicking Performance in Young U9 to U20 Soccer Players: Assessment of Velocity and Accuracy Simultaneously
}

\author{
Luiz H. P. Vieira $\left(\mathbb{1},{ }^{1}\right.$ Sérgio A. Cunha $\left(\mathbb{0},{ }^{2}\right.$ Renato Moraes $\left(\mathbb{0},{ }^{1}\right.$ Fabio A. Barbieri $\left(\mathbb{1}^{3},{ }^{3}\right.$ Rodrigo Aquino $\left(\mathbb{D},{ }^{1,4}\right.$ Lucas de
} P. Oliveira (10, ${ }^{1}$ Martina Navarro $\mathbb{B}^{5,},{ }^{5,6}$ Bruno L. S. Bedo (10, ${ }^{1}$ and Paulo R. P. Santiago (1) ${ }^{1}$

'University of São Paulo; ${ }^{2}$ UNICAMP; ${ }^{3}$ São Paulo State University; ${ }^{4}$ University of Porto; ${ }^{5}$ Federal University of São Paulo; ${ }^{6}$ University of Bern

\begin{abstract}
Purpose: The purpose of this study was to compare the kicking performance of young soccer players in the U9 to U20 age groups. Method: Three hundred and sixty-six Brazilian players were evaluated on an official pitch using three-dimensional kinematics to measure $(300 \mathrm{~Hz})$ ball velocity $\left(V_{\text {ball }}\right)$, foot velocity $\left(V_{\text {foot }}\right), V_{\text {ball }} / V_{\text {foot }}$ ratio, last stride length, and distance between the support foot and the ball. Simultaneously, a two-dimensional procedure was also conducted to compute $(60 \mathrm{~Hz})$ the mean radial error, bivariate variable error, and accuracy. Possible age-related differences were assessed through one-way analysis of variance and magnitude-based inferences. Results: Ball velocity increased by $103 \%\left(p<.001, \eta^{2}=.39\right)$ from the U11 age group $(48.54 \pm 8.31 \mathrm{~km} / \mathrm{hr})$ to the U20 age group $(98.74 \pm 16.35 \mathrm{~km} / \mathrm{hr})$. Foot velocity presented a $59 \%$ increase $\left(p<.001, \eta^{2}=.32\right)$ from the U11 age group $(49.08 \pm 5.16 \mathrm{~km} / \mathrm{hr})$ to U20 (78.24 $\pm 9.49 \mathrm{~km} / \mathrm{hr}$ ). This finding was due to improvement in the quality of foot-ball impact $\left(\mathrm{V}_{\text {ball }} / \mathrm{V}_{\text {foot }}\right.$ ratio) from U11 (0.99 \pm 0.13 a.u.) to U20 (1.26 \pm 0.11 a.u.; $\left.p<.001, \eta^{2}=.25\right)$. Parameters such as mean radial error and accuracy appeared to be impaired during the growth spurt (U13U15). Last stride length was correlated, low to moderately high, with $\mathrm{V}_{\text {ball }}$ in all age groups $(r=.36-.79)$. Conclusions: In summary, we concluded that simple biomechanical parameters of kicking performance presented distinct development. These results suggest that different training strategies specific for each age group could be applied. We provide predictive equations to aid coaches in the long-term monitoring process to develop the kick in soccer or search for talented young players.
\end{abstract}

\section{ARTICLE HISTORY}

Received 28 July 2017

Accepted 7 February 2018

\section{KEYWORDS}

Association football; development; technical skill; three-dimensional kinematics
Development of the computational sciences (e.g., computer vision and image processing) has improved the analysis techniques and measurement systems applied to human movement research. These advances have allowed for expansion of the knowledge about threedimensional (3D) kinematic and kinetic characteristics in soccer kicking (Lees, Asai, Andersen, Nunome, \& Sterzing, 2010). Kicking performance has been widely reported in previous studies with youth players (Anderson \& Sidaway, 1994; Barnett, van Beurden, Morgan, Brooks, \& Beard, 2010; Berjan Bacvarevic et al., 2012; Juarez, Lopez de Subijana, Mallo, \& Navarro, 2011; Kapidzic, Huremovic, \& Biberovic, 2014; Katis, Kellis, \& Lees, 2015; Wilson et al., 2016; Wong, Chamari, Dellal, \& Wisloff, 2009), varying in age from 6 years (Teixeira \& Teixeira, 2008) to 20 years old (Apriantono, Nunome, Ikegami, \& Sano, 2006). However, only a limited number of works have aimed to verify the effects of age on direct measures of kicking performance, and these studies have included a small range of age groups (Berjan Bacvarevic et al., 2012; Cerrah, Şimşek, Soylu, Ertan, \& Nunome, 2015; Katis et al., 2015; Teixeira \& Teixeira, 2008).

Kicking is the defining action in soccer (Lees et al., 2010), and searching for performance indicators that help to achieve success in this skill is one of the most commonly raised issues of applied biomechanics in soccer. Ball velocity and accuracy are considered the main factors that contribute to a successful kicking outcome (van den Tillaar \& Ulvik, 2014; Vieira et al., 2016). In the past, it has been shown that short-term training (10 weeks) may be sufficient to achieve a typical pattern of proximal-distal coordination in improving kicking performance, but only in young adults (Anderson \& Sidaway, 1994). In recent years, the process of talent identification and development has become a very important issue in the soccer context and research. Yet, the results obtained in studies that have focused on clarifying the evolution of kicking performance with advancement in chronological age

CONTACT Paulo R. P. Santiago paulosantiago@usp.br $\otimes$ School of Physical Education and Sport of Ribeirão Preto, University of São Paulo, Av. Bandeirantes 3900, Monte Alegre-Ribeirão Preto/SP, 14040-907, Brazil.

(c) 2018 SHAPE America 
are still discordant. For instance, some studies have shown that kicking is not an age-dependent skill, especially in terms of accuracy, which does not demonstrate change with age (Malina et al., 2005; Malina, Ribeiro, Aroso, \& Cumming, 2007; Rosch et al., 2000; Vaeyens et al., 2006). A different framework was noted when analyzing ball velocity, for which increases were found (Berjan Bacvarevic et al., 2012; Cerrah et al., 2015; Katis et al., 2015).

An important literature review on this topic pointed to the existence of a gap between research on sports biomechanics and the teaching-learning-training process (Lees et al., 2010). In this sense, velocity and accuracy were not considered simultaneously in the majority of studies that we reviewed. These parameters have been analyzed in isolation in youth soccer (Juarez et al., 2011; Kapidzic et al., 2014; Katis et al., 2015; Malina et al., 2005, 2007; Rosch et al., 2000; Teixeira \& Teixeira, 2008; Vaeyens et al., 2006; Wilson et al., 2016; Wong et al., 2009). The prioritization or selection of only one parameter (e.g., velocity) may represent a significant loss of the other parameter (e.g., accuracy), as shown in a previous study that revealed a speedaccuracy trade-off when kicking with the dominant limb (van den Tillaar \& Ulvik, 2014). Also, many authors have used different types of technology (e.g., speed radar, Berjan Bacvarevic et al., 2012; Wong et al., 2009; videogrammetry, Juarez et al., 2011; Kapidzic et al., 2014; Katis et al., 2015; Teixeira \& Teixeira, 2008; and microphones, Navarro, van der Kamp, Ranvaud, \& Savelsbergh, 2013). Low acquisition frequency to investigate the kicking task has been an important limitation in some previous work (e.g., $60 \mathrm{~Hz}$, Anderson \& Sidaway, 1994; Orloff et al., 2008; Teixeira \& Teixeira, 2008). In addition, radars provide limited information about lower-limb movements. Target size and location, type of kick performed, ball used, footwear, collection environment, number of trials, and instructions were not standardized among previous studies. It is difficult to directly compare the data from previous cross-sectional studies in youth players to understand performance evolution as a function of chronological age.

Young players from different countries on different continents around the world and with distinct mean ages have already been evaluated (e.g., Asia, Japan [20 years; Apriantono et al., 2006], China [U14; Wong et al., 2009]; Oceania, Australia [16.8 years; McLean \& Tumilty, 1993]; Europe, Spain [16.1 years; Juarez et al., 2011], Turkey [12-17 years; Cerrah et al., 2015], The Netherlands [19.1 years; Navarro et al., 2013], Bosnia [13 years; Kapidzic et al., 2014], Serbia [12.2-15.3 years; Berjan Bacvarevic et al., 2012]; and North America,
United States [20.2 years; Orloff et al., 2008], uninformed locations [e.g., 15.1 years; Katis et al., 2015]). Geographic location may contribute to possible differences, depending on the models of introduction to the sport, training process, and specialization (Vieira et al., 2017). For instance, when analyzing maximal kicks in age-matched players (13 years old), Kapidzic et al. $(2014 ; \sim 75.42 \pm 8.86 \mathrm{~km} / \mathrm{hr}$ ) and Berjan Bacvarevic et al. $(2012 ; \sim 89.68 \pm 6.64 \mathrm{~km} / \mathrm{hr})$ found different mean ball velocities. Data on the population of young South American players are still unknown. Understanding the development of kicking could have practical applications for talent identification and training prescription, and it could reveal critical and sensitive periods to develop specific performance parameters. Thus, the purpose of this study was to compare kick performance in young trained soccer players in the U9 to U20 age groups from a large sample. Our hypothesis was that increases in some kicking performance parameters would be foundmainly velocities (ball velocity, foot velocity, and the ratio between them)-while the error rates (accuracy measures) would simultaneously show decreases with age advance.

\section{Methods}

\section{Experimental design}

The experimental protocol was conducted on an official pitch with natural grass (FIFA standard, $100 \mathrm{~m} \times 70 \mathrm{~m}$; goal dimensions, $7.32 \mathrm{~m} \times 2.44 \mathrm{~m})$ in the presence of sunlight (9:00 a.m.-10:30 a.m. and 4:00 p.m.5:30 p.m.). Data were collected from June 2014 to December 2016. All players wore the equipment (i.e., footwear and clothing) usually adopted for training sessions and official soccer competitions. First, the anthropometric parameters were measured: weight (digital scale Sb623, DLK Sports ${ }^{\oplus}$, São Paulo, Brazil; sensitivity $=0.1 \mathrm{~kg}$ ), height (PRIME MED stadiometer, São Paulo, Brazil; sensitivity $=0.1 \mathrm{~cm}$ ), and time to peak height velocity $(\mathrm{PHV}=-7.999994+[0.0036124 \times$ $($ Age $\times$ Height)]; Moore et al., 2015). Prior to the kick protocol, participants performed a general warm-up of $10 \mathrm{~min}$, composed of moderate-intensity running, ballistic stretching, and specific kick exercises to warm the body and avoid injury during the test phase (van den Tillaar \& Ulvik, 2014; Vieira et al., 2017). Each participant then performed penalty kicks using the dominant lower limb, with a distance of $11 \mathrm{~m}$ between the initial ball position and goal plane, to hit a $1-\mathrm{m} \times 1-\mathrm{m}$ target (Figure $1[\mathrm{C}]$ ) positioned in the goal center (van den Tillaar \& Ulvik, 2014). Identification of the dominant 


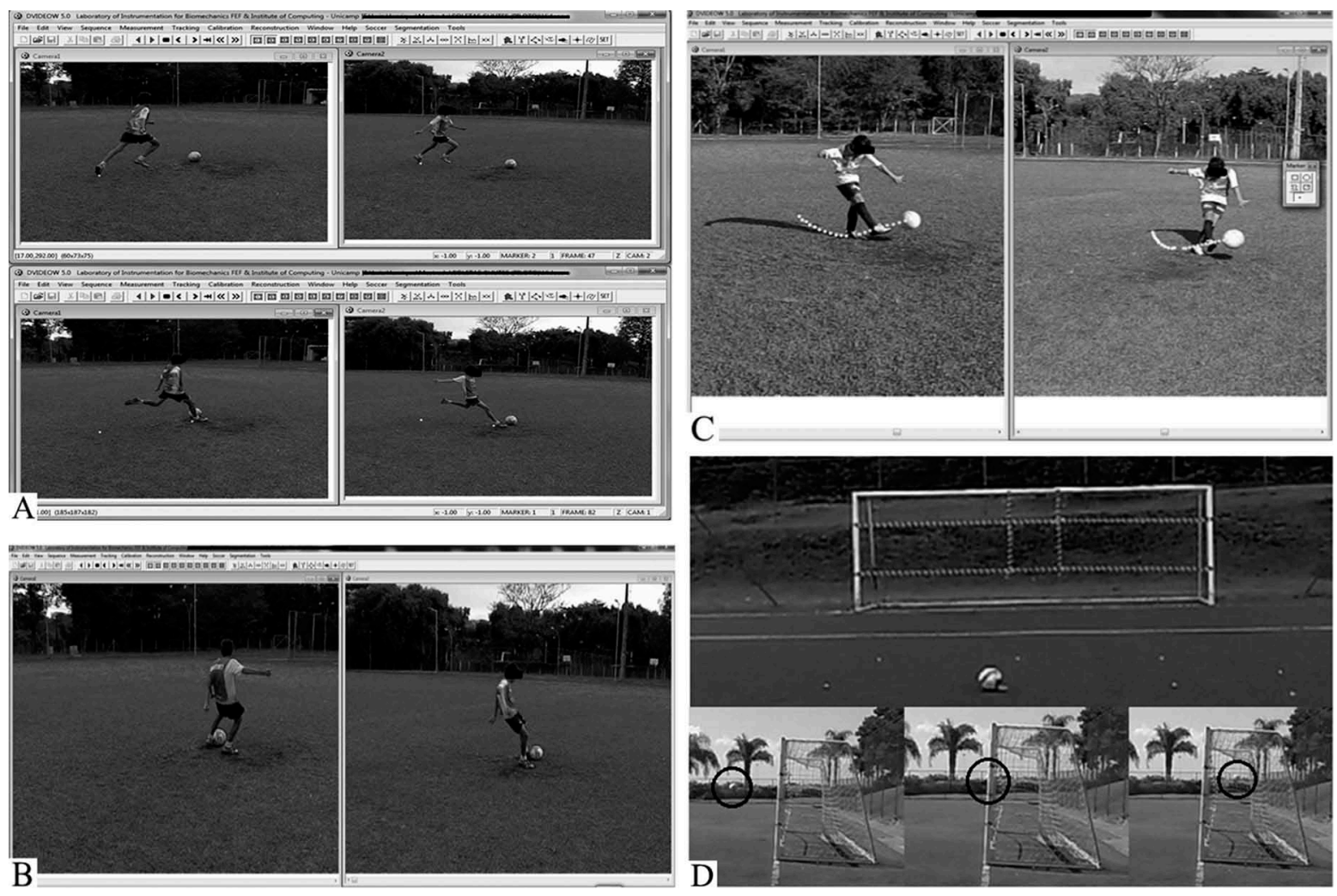

Figure 1. Experimental setup, including target illustration, to obtain (A) the last stride length, (B) distance between support foot and ball, (C) foot velocity and ball velocity, and (D) view of the camera that recorded the distance between the ball centroid and the center of the target and view of the lateral camera, which detected when the ball crossed goal line.

lower limb was obtained by self-report (Vieira et al., 2016). Participants were instructed to "kick with maximum ball velocity as possible and hit the center of the target" (Apriantono et al., 2006; Milioni et al., 2016; van den Tillaar \& Ulvik, 2014; Vieira et al., 2016), with an instep kick type. Standardized balls (PENALTY ${ }^{\circledR}$ brand, FIFA-approved) were used according to the norms of the São Paulo Football Federation (Brazil). Regarding dimensions and weight for each age group, Size 4 was used in the U9 and U11 groups $(65 \mathrm{~cm} \varnothing, 375 \mathrm{~g})$ and Size 5 was used for the U13 to U20 groups $(70 \mathrm{~cm} \varnothing$, $430 \mathrm{~g}$ ). Each participant performed three trials (Berjan Bacvarevic et al., 2012; Vieira et al., 2016), and no restrictions were imposed on the number of steps, approach angle, or interval, which were autoselected (Barbieri, Gobbi, Santiago, \& Cunha, 2015; Lees, Kershaw, \& Moura, 2005).

\section{Participants}

Three hundred and sixty-six $(N=366)$ young male Brazilian soccer players participated in this study. Participants were included in specific groups according to their age: U9 (means \pm standard deviations, $n=11$, $8.6 \pm 1.1$ years, $30.1 \pm 5.7 \mathrm{~kg}, 131.3 \pm 4.4 \mathrm{~cm}$; $\mathrm{PHV}=-4.02 \pm 0.35), \mathrm{U} 11(n=73,10.4 \pm 0.5$ years, $38 \pm 5.3 \mathrm{~kg}, 149.6 \pm 6.3 \mathrm{~cm}, \mathrm{PHV}=-2.19 \pm 0.41), \mathrm{U} 13$ $(n=80,12.3 \pm 0.5$ years, $50.8 \pm 10.7 \mathrm{~kg}, 160.2 \pm 9.6 \mathrm{~cm}$, $\mathrm{PHV}=-0.70 \pm 0.85), \mathrm{U} 15(n=105,14.2 \pm 0.6$ years, $62.6 \pm 9.2 \mathrm{~kg}, 173.5 \pm 8.7 \mathrm{~cm}, \mathrm{PHV}=1.23 \pm 0.44), \mathrm{U} 17$ $(n=78,15.9 \pm 0.6$ years, $66.9 \pm 6.1 \mathrm{~kg}, 175.8 \pm 7 \mathrm{~cm}$, $\mathrm{PHV}=1.49 \pm 0.81)$, and $\mathrm{U} 20(n=19,18.4 \pm 0.9$ years, $74.5 \pm 6.9 \mathrm{~kg}, 180.6 \pm 5.4 \mathrm{~cm}, \mathrm{PHV}=3.48 \pm 0.89$ ). To participate in this study, participants were required to have initiated formal training at the age of 6 years old (Teixeira \& Teixeira, 2008). Participants were excluded if they had a professional contract to train and/or compete at their age level. All procedures were approved by the local human research ethics committee in accordance with the Code of Ethics of the World Medical Association (approved by the Ethics Advisory Board of Swansea University). All participants signed a written consent form confirming in writing their participation as a volunteer, and players aged younger than 18 years old only participated if their guardians also signed a consent form. Players in the U9 through U13 age groups participated in 3 days of training per week 
(one conditioning session of stretching and running drills without the ball, one to two sessions of isolated technical skills, and two specific sessions including simulated matchplay and technical-tactical exercises such as small-sided, conditioned games and game subphases [e.g., $1 \mathrm{v} 1+$ goalkeeper]). The U15 to U20 age groups participated in 4 days to 5 days of training per week with one to two conditioning sessions without the ball (focused on stretching, running, and strength development), one session of isolated technical skills, and three to four specific sessions in the field (similar content to that described for younger players). All players trained for approximately $90 \mathrm{~min}$ per day and participated in one competition match on the weekends.

\section{Kinematic procedures}

The experimental protocol was monitored by four digital video cameras positioned at specific points on the field. Two cameras (Casio Exilim Highspeed EX-F1, CASIO ${ }^{\circ}$ Computer Co. Ltd., Tokyo, Japan) were allocated laterally (Figure $1[\mathrm{~A}]$ ), with a distance of $3 \mathrm{~m}$ from the penalty mark, forming an angle of $90^{\circ}$ between them and $45^{\circ}$ with the ball adjusted to an acquisition frequency of $300 \mathrm{~Hz}$ (National Television System Committee [NTSC] standard; shutter speed of 1/2,000 s, 512-pixel $\times 384$ pixel resolution). For the spatial calibration, an orthogonal triad $(1 \mathrm{~m} \times 1 \mathrm{~m} \times 1 \mathrm{~m})$ containing 10 markers with known absolute positions on the $x, y$, and $\mathrm{z}$ axes (Milioni et al., 2016) was used. The $\mathrm{z}$ axis was defined as vertical (pointing up), the y axis was anteroposterior (pointing to the center of the goal and orthogonal to $\mathrm{z}$ ), and the $\mathrm{x}$ axis was obtained by the vector product of $y$ by $z$ (pointing laterally). The sequences were transferred to the computer, and the commands were run (Barbieri et al., 2015; Milioni et al., 2016; Vieira et al., 2016) for (a) synchronization by a beep that was issued when all the cameras were turned on, (b) a calibration frame definition, (c) frame marking (Figure $1[\mathrm{~A}]-$ Figure $1[\mathrm{C}]$ ), and (d) 3D direct linear transformation reconstruction using DVIDEOW software (Campinas State University, Campinas/São Paulo, Brazil; Figueroa, Leite, \& Barros, 2003). A data matrix containing the $3 \mathrm{D}$ spatial coordinates of each measured marker was obtained for each shot performed $(N=1,098$ trials). An accuracy test was carried out (Barbieri et al., 2015; Milioni et al., 2016; Vieira et al., 2016), and the experimental uncertainties for this study were low (precision $=1 \mathrm{~cm}$, bias $=0.9 \mathrm{~cm}$, accuracy $=1.4 \mathrm{~cm}$ ).

\section{Dependent variables}

The following dependent variables were calculated for each trial: last stride length (LSL; Kapidzic et al., 2014; Lees et al., 2005), which was defined as the Euclidean distance between the place where the dominant foot lost contact with the ground and the calcaneus of the nondominant foot when it landed on the ground during the last stride; distance between the support foot and the ball

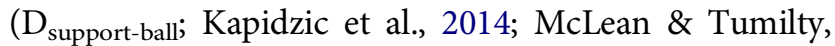
1993; Orloff et al., 2008), which was defined as the Euclidean distance between the centroid of the support foot and the centroid of the ball at the instant of impact; foot velocity ( $\mathrm{V}_{\text {foot}}$; Barbieri et al., 2015; Juarez et al., 2011), which was measured from the displacement of the marker on the distal phalanx of the fifth metatarsus of the dominant foot; and ball velocity ( $\mathrm{V}_{\text {ball }}$; Katis et al., 2015; Milioni et al., 2016), which was measured from the displacement of the ball centroid (Figure $1[\mathrm{C}]$ ). We also computed the $\mathrm{V}_{\text {ball }} / \mathrm{V}_{\text {foot }}$ ratio as a dependent measure. Data treatment and calculations were conducted using written routines in the Matlab ${ }^{\star}$ environment (The MathWorks Inc., Natick, MA, USA). Kinematic data (i.e., fifth metatarsal trajectory) were filtered by the locally weighted smoothing quadratic fit function (Barbieri et al., 2015) with a smoothing parameter (span $=0.7)$ selected after residual analysis. To smooth the ball trajectory, the horizontal components were considered linear and were calculated as the first derivative of the linear regression lines, adjusted to their unfiltered displacements. The vertical component was considered to be the second degree and was calculated as the first derivative of a quadratic regression line with its second derivative set as equal to $-9.8 \mathrm{~m}^{-1}$ and adjusted to its nonfiltered displacement in the airborne frames. Ten frames were considered before contact with the ball to calculate the average $3 \mathrm{D} \mathrm{V}_{\text {foot }}$, and 10 frames were considered after foot contact with the ball to calculate the average 3D $\mathrm{V}_{\text {ball }}$ (Barbieri et al., 2015; Milioni et al., 2016; Vieira et al., 2016).

\section{Mean radial error, bivariate variable error, and accuracy}

Two auxiliary cameras (GoPro ${ }^{\circledR}$ Hero 3+ Black Edition, Woodman Labs Inc., San Mateo, CA, USA) were used to determine kicking accuracy. One was placed frontally, $17 \mathrm{~m}$ from the goal line, and the other was placed at the intersection of the goal line with the goalkeeper's area line to identify the moment the ball crossed the goal line (Figure 1[D]). Both cameras were set at $60 \mathrm{~Hz}$ (superview mode 1,920-pixel $\times 1,080$-pixel resolution, NTSC standard; Milioni et al., 2016). The radial 
distortion was previously corrected (Vieira et al., 2016). Next, the ball centroid at the moment it crossed the goal line was digitized in two-dimensional (2D) by an operator, similar to the kinematic procedures and using the same software. By means of specific routines, the distance from the ball to the center of the target $\left(D_{\text {target }}\right.$; Barbieri et al., 2015) was calculated, and from this value, the dependent variables of mean radial error (MRE), bivariate variable error (BVE), and accuracy (ACUR; Vieira et al., 2016) were calculated according to the following Equations (1)-(4):

$$
\begin{gathered}
D_{\text {target }}=\sqrt{\left(x_{\text {target }}-x_{\text {ball }}\right)^{2}+\left(y_{\text {target }}-y_{\text {ball }}\right)^{2}} \\
M R E=\frac{1}{N} \sum_{i=1}^{N}\left|D_{\text {target }}\right| \\
B V E=\sqrt{\left(S D_{x-\text { coordinate }}\right)^{2}+\left(S D_{y \text {-coordinate }}\right)^{2}} \\
A C U R=\sqrt{(M R E)^{2}+(B V E)^{2}}
\end{gathered}
$$

where $D_{\text {target }}=$ Euclidean distance between the ball centroid and target center; $(x, y)_{\text {target }}=2 \mathrm{D}$ coordinates of the target center point; $(x, y)_{\text {ball }}=2 \mathrm{D}$ coordinates of the ball centroid when it crosses the goal line; $N=$ number of trials; $S D_{x-\text { coordinate }}=$ standard deviation of $x_{\text {ball }}$ coordinates in the three trials; $S D_{y \text {-coordinate }}=$ standard deviation of $y_{\text {ball }}$ coordinates in the three trials.

\section{Statistical analysis}

The normal distribution of the data was verified using the Kolmogorov-Smirnov test. The data are presented as means \pm standard deviations and confidence intervals (95\%). The one-way analysis of variance (ANOVA), followed by the Tukey-Kramer post-hoc, was used to compare kicking performance between age groups. The Pearson's product-moment correlation test was used to verify possible associations between $\mathrm{V}_{\text {ball }}$, age, and all other dependent variables. Linear regressions were performed to estimate the contribution of chronological age to the variation in kicking performance $\left(R^{2}\right)$. The significance level was set at $p<.05$. For each ANOVA, the partial eta squared $\left(\eta^{2}\right)$ was calculated. For all comparisons, we also calculated the Cohen's $d$. Effect size limit values were $d>0.2$ (small), $>0.5$ (medium), and $>0.8$ (large); $\eta^{2}>.01$ (small), > .06 (medium), and > .15 (large); and $R^{2}>.02$ (small), > .13 (medium), and > .26 (large; Cohen, 1988).
Using a 95\% confidence interval, we also calculated whether the chances of true differences (unknown) between two groups exhibiting lower, similar, or higher values (i.e., higher than the smallest practically important effect, or smallest worthwhile change $[0.2$ multiplied by the between-subject deviation, based on the Cohen's $d$ principle]). The quantitative chance (QC) was interpreted as: $\leq 1 \%=$ almost certainly not, $>1 \%$ to $5 \%=$ very unlikely, $>5 \%$ to $25 \%=$ unlikely, $>25 \%$ to $75 \%=$ possibly, $>75 \%$ to $95 \%=$ likely, $>95 \%$ to $99 \%=$ very likely, $>99 \%=$ almost certain. If the likelihood of higher or lower differences was $>5 \%$ for both, the true difference was reported as unclear. Otherwise, we interpreted this change as the observed difference (Hopkins, Marshall, Batterham, \& Hanin, 2009). Statistical analyses were performed on IBM Statistical Package for the Social Sciences (SPSS) Statistics Version 20 (Armonk, NY) and Microsoft Excel spreadsheets (available at http://www.sportsci.org).

\section{Results}

In Table 1, the results for all variables are presented according to age group. The ANOVA revealed that $\mathrm{V}_{\text {ball }}$ was significantly different, $F(5,360)=45.49$, $p<.001, \eta^{2}=.39$, between age groups. Post-hoc comparisons indicated statistical differences between age groups (U20 > U17, U15 > U13 > U11, U9; 100/0/0 almost certain, $d=0.71-3.87)$, except between the U9 and U11 age groups ( $p=.33,75 / 17 / 8$ unclear, $d=0.96$ ) and between the U15 and U17 age groups $(p=.29,97 /$ $3 / 0$ likely, $d=0.28$ ). Statistical analysis for the $\mathrm{V}_{\text {foot }}$ identified significant differences between groups, similar to what was observed for the $\mathrm{V}_{\text {ball }}(\mathrm{U} 20>\mathrm{U} 17, \mathrm{U} 15$ $>\mathrm{U} 13>\mathrm{U} 11, \mathrm{U} 9 ; d=0.76-3.82)$. Significant differences were found, $F(5,360)=34.03, p<.001, \eta^{2}=.32$, with increases as a function of age. Again, the absence of differences occurred between the U9 and U11 age groups $(p=.66,92 / 6 / 2$ likely, $d=0.75)$ and the U15 and U17 age groups $(p=.22,66 / 34 / 0$ possibly, $d=0.76)$. The QCs were almost certain for the majority of comparisons (100/0/0) except for U17 versus U20 (98/2/0 very likely, $d=0.91)$ and those comparisons mentioned. Similarly, the $\mathrm{V}_{\text {ball }} / \mathrm{V}_{\text {foot }}$ ratio presented progressive increases, $F(5,360)=24.23, p<.001$, $\eta^{2}=.25$, from U9 to U20. However, in the post-hoc comparisons, some near age groups did not differ significantly. This was the case for U9 versus U11 ( $p=.12$, $54 / 35 / 11$ unclear, $d=0.66)$, U11 versus U13 ( $p=.64$, $55 / 44 / 1$ possibly, $d=0.27$ ), U15 versus U17 ( $p=.98,25 /$ $72 / 4$ unclear, $d=0.1)$, and U17 versus U20 ( $p=.09,67 /$ $28 / 5$ unclear, $d=0.67)$. Remaining comparisons were likely to almost certain $(d=0.76-2.24)$. 
Table 1. Mean \pm standard deviation (95\% confidence limits) for kicking performance variables according to age $(n=366)$.

\begin{tabular}{|c|c|c|c|c|c|c|}
\hline & U9 & U11 & U13 & U15 & U17 & U20 \\
\hline $\mathrm{V}_{\text {ball }}\left(\mathrm{km} / \mathrm{hr}^{-1}\right)$ & $48.54 \pm 8.31^{\mathbf{a}, \mathbf{b}, \mathbf{c}, \mathbf{d}}$ & $57.87 \pm 10.93^{\mathbf{e}, \mathbf{f}, \mathbf{g}, \mathbf{h}}$ & $66.70 \pm 13^{\mathbf{i}, \mathbf{j}, \mathbf{k}}$ & $76.92 \pm 15.58^{1}$ & $81.35 \pm 16.04^{\mathrm{m}}$ & $98.74 \pm 16.35$ \\
\hline $\mathrm{V}_{\text {foot }}\left(\mathrm{km} / \mathrm{hr}^{-1}\right)$ & $\begin{array}{c}{[42.96,54.12]} \\
49.08 \pm 5.16^{\mathbf{a}, \mathbf{b}, \mathbf{c}, \mathbf{d}} \\
{[45.62,52.55]}\end{array}$ & $\begin{array}{c}{[55.32,60.42]} \\
53.79 \pm 7.25^{\mathbf{e}, \mathbf{f}, \mathbf{g}, \mathbf{h}} \\
{[52.10,55.48]}\end{array}$ & $\begin{array}{c}{[63.81,69.60]} \\
60.54 \pm 8.77^{\mathbf{i}, \mathbf{j}, \mathbf{k}} \\
{[58.58,62.49]}\end{array}$ & $\begin{array}{l}{[73.90,79.93]} \\
65.17 \pm 10.43^{1} \\
{[63.15,67.19]}\end{array}$ & $\begin{array}{c}{[77.74,84.97]} \\
68.44 \pm 11.83^{\mathrm{m}} \\
{[65.77,71.10]}\end{array}$ & $\begin{array}{c}{[90.55,106.62]} \\
78.24 \pm 9.49 \\
{[73.66,82.81]}\end{array}$ \\
\hline $\mathrm{V}_{\text {ball }} / \mathrm{V}_{\text {foot }}$ ratio (a.u.) & $\begin{array}{c}0.99 \pm 0.13^{\mathbf{a}, \mathbf{b}, \mathbf{c}, \mathbf{d}} \\
{[0.90,1.07]}\end{array}$ & $\begin{array}{c}1.07 \pm 0.11^{\mathbf{f}, \mathbf{g}, \mathbf{h}} \\
{[1.05,1.10]}\end{array}$ & 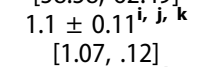 & $\begin{array}{l}1.18 \pm 0.1^{1} \\
{[1.16,1.20]}\end{array}$ & $\begin{array}{l}1.19 \pm 0.1 \\
{[1.16,1.21]}\end{array}$ & $\begin{array}{l}1.26 \pm 0.11 \\
{[1.21,1.31]}\end{array}$ \\
\hline LSL (m) & $\begin{array}{c}1.09 \pm 0.14^{\mathbf{a}, b}, \mathbf{b}, \mathbf{d} \\
{[1.00,1.18]}\end{array}$ & $\begin{array}{c}1.25 \pm 0.14^{\mathbf{e}, \mathbf{f}, \mathbf{g}, \mathbf{h}} \\
{[1.21,1.28]}\end{array}$ & $\begin{array}{c}1.36 \pm 0.19^{\mathbf{k}} \\
{[1.32,1.40]}\end{array}$ & $\begin{array}{l}1.43 \pm 0.23^{1} \\
{[1.38,1.47]}\end{array}$ & $\begin{array}{l}1.44 \pm 0.2^{\mathrm{m}} \\
{[1.39,1.48]}\end{array}$ & $\begin{array}{c}1.6 \pm 0.14 \\
{[1.54,1.67]}\end{array}$ \\
\hline $\mathrm{D}_{\text {support-ball }}(\mathrm{m})$ & $\begin{array}{l}0.33 \pm 0.07 \\
{[0.28,0.37]}\end{array}$ & $\begin{array}{c}0.3 \pm 0.07^{\mathbf{e}, \mathbf{f}, \mathbf{g}} \\
{[0.29,0.32]}\end{array}$ & $\begin{array}{l}0.34 \pm 0.06 \\
{[0.33,0.35]}\end{array}$ & $\begin{array}{l}0.34 \pm 0.09 \\
{[0.33,0.36]}\end{array}$ & $\begin{array}{l}0.34 \pm 0.06 \\
{[0.33,0.36]}\end{array}$ & $\begin{array}{l}0.35 \pm 0.04 \\
{[0.32,0.37]}\end{array}$ \\
\hline MRE (m) & $\begin{array}{l}1.4 \pm 0.49 \\
{[1.07,1.73]}\end{array}$ & $\begin{array}{c}1.65 \pm 0.6^{\mathbf{f}, \mathbf{g}, \mathbf{h}} \\
{[1.52,1.79]}\end{array}$ & $\begin{array}{c}1.59 \pm 0.59^{\mathbf{i}, \mathbf{j}, \mathbf{k}} \\
{[1.46,1.72]}\end{array}$ & $\begin{array}{l}1.34 \pm 0.48 \\
{[1.24,1.43]}\end{array}$ & $\begin{array}{l}1.29 \pm 0.5 \\
{[1.18,1.40]}\end{array}$ & $\begin{array}{l}1.14 \pm 0.35 \\
{[0.98,1.31]}\end{array}$ \\
\hline BVE (m) & $\begin{array}{l}1.26 \pm 0.58 \\
{[0.87,1.65]}\end{array}$ & $\begin{array}{c}1.47 \pm 0.73^{\mathbf{f}, \mathbf{g}, \mathbf{h}} \\
{[1.31,1.64]}\end{array}$ & $\begin{array}{l}1.30 \pm 0.57 \\
{[1.17,1.43]}\end{array}$ & $\begin{array}{l}1.18 \pm 0.51 \\
{[1.08,1.27]}\end{array}$ & $\begin{array}{l}1.17 \pm 0.5 \\
{[1.06,1.28]}\end{array}$ & $\begin{array}{l}1.05 \pm 0.32 \\
{[0.90,1.21]}\end{array}$ \\
\hline ACUR (m) & $\begin{array}{l}1.93 \pm 0.64 \\
{[1.50,2.36]}\end{array}$ & $\begin{array}{c}2.25 \pm 0.84^{\mathbf{f}, \mathbf{g}, \mathbf{h}} \\
{[2.06,2.45]}\end{array}$ & $\begin{array}{l}2.09 \pm 0.72 \\
{[1.93,2.25]}\end{array}$ & $\begin{array}{l}1.81 \pm 0.62 \\
{[1.69,1.93]}\end{array}$ & $\begin{array}{c}1.77 \pm 0.5 \\
{[1.63,1.92]}\end{array}$ & $\begin{array}{c}1.57 \pm 0.4 \\
{[1.38,1.76]}\end{array}$ \\
\hline
\end{tabular}

Note. $\quad \mathrm{a}=\mathrm{U} 9 \times \mathrm{U} 13 ; \mathrm{b}=\mathrm{U} 9 \times \mathrm{U} 15 ; \mathrm{c}=\mathrm{U} 9 \times \mathrm{U} 17 ; \mathrm{d}=\mathrm{U} 9 \times \mathrm{U} 20 ; \mathrm{e}=\mathrm{U} 11 \times \mathrm{U} 13 ; \mathrm{f}=\mathrm{U} 11 \times \mathrm{U} 15 ; \mathrm{g}=\mathrm{U} 11 \times \mathrm{U} 17 ; \mathrm{h}=\mathrm{U} 11 \times \mathrm{U} 20 ; \mathrm{i}=\mathrm{U} 13 \times \mathrm{U} 15 ; \mathrm{j}=\mathrm{U} 13 \times$ $\mathrm{U} 17 ; \mathrm{k}=\mathrm{U} 13 \times \mathrm{U} 20 ; \mathrm{I}=\mathrm{U} 15 \times \mathrm{U} 20 ; \mathrm{m}=\mathrm{U} 17 \times \mathrm{U} 20$. Confidence limits $=$ (lower, upper bound). $\mathrm{V}_{\text {ball }}=$ ball velocity; $\mathrm{V}_{\text {foot }}=$ foot velocity; $\mathrm{V}_{\text {ball }} / \mathrm{V}_{\text {foot }}=$ ball velocity-to-foot velocity ratio; $L S L=$ last stride length; $D_{\text {support-ball }}=$ distance between support foot and ball; $M R E=$ mean radial error; $B V E=$ bivariate

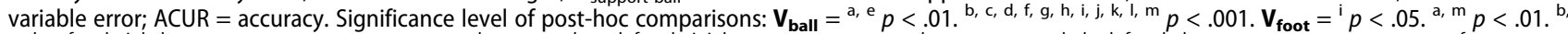

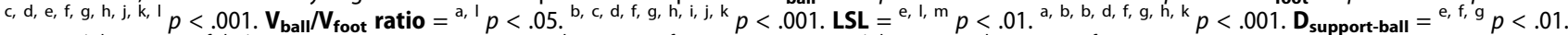
MRE $={ }^{\mathrm{i}, \mathrm{k}} p<.05 .{ }^{\mathrm{f}, \mathrm{h}, \mathrm{j}} p<.01 .{ }^{\mathrm{g}} p<.001 . \mathbf{B V E}={ }^{\mathrm{g}, \mathrm{h}} p<.05 .{ }^{\mathrm{f}} p<.01$. ACUR $={ }^{\mathrm{j}, \mathrm{k}} p<.05 .{ }^{\mathrm{h}} p<.01 .{ }^{\mathrm{f}, \mathrm{g}} p<.001$.

The LSL also presented differences, $F(5,360)=19.92$, $p<.001, \eta^{2}=.22$, which suggested increases as a function of age. Some groups, generally near, did not present significant statistical differences, although QCs were observed. This was the case for the post-hoc comparisons between the U9 and U11 age groups ( $p=.13,99 / 1 / 0$ very likely, $d=1.14$ ) and U13 and U15 age groups ( $p=.16,86 / 14 / 0$ likely, $d=0.33)$. In addition, supporting the significant statistical differences verified, very likely QCs $(98 / 2 / 0$, $d=0.89-1.62)$ were identified between the U9 and U13 age groups, U13 and U20 age groups, U15 and U20 age groups, and U17 and U20 age groups. In contrast, a likely QC (91/9/0) between the U13 and U17 was obtained. Finally, there were no differences between the U15 and U17 age groups ( $p=1,23 / 72 / 5$ unclear, $d=0.05)$. The $\mathrm{D}_{\text {support-ball }}$ presented a very distinct pattern of development (see Table 1). Although the ANOVA pointed to an effect of age group, $F(5,360)=4.30, p=.001, \eta^{2}=.06$, only differences between the U11 and other age groups were observed ( $d=0.5-0.61$; i.e., vs. U13 [99/1/0 very likely], U15 [93/7/0 likely], and U17 [99/1/0 very likely]). In the comparison between U9 ( $p=.91,13 / 28 / 59$ unclear) and U20 $(p=.11,62 / 32 / 6$ unclear), the difference was not identified $(d=0.42-0.88)$. The other post-hoc comparisons, in addition to being nonsignificant, were unclear $(d \leq 0.35)$.

Mean radial error, $F(5,360)=6.92, p<.001$, $\eta^{2}=.09$, was greater for the U11 group $(d=0.57-$ 1.04) than for the U15 ( $p=.001,0 / 4 / 96$ very likely), $\mathrm{U} 17$ ( $p<.001,0 / 0 / 100$ almost certain), and U20 ( $p=.003,0 / 1 / 99$ very likely) groups. The U13 group also presented higher indices $(d=0.46-0.93)$ than the $\mathrm{U} 15$ ( $p=0.017,0 / 3 / 97$ very likely), $\mathrm{U} 17(p=.006,0 / 2 /$ 98 very likely), and $\mathrm{U} 20$ ( $p=0.012,1 / 11 / 88$ likely $)$ groups. Other QCs were unclear $(d=0.1-0.61)$, except between the U15 and U20 groups (1/14/85 likely, $d=0.48)$. With regard to the BVE, $F(5,360)=3.55$, $p=.004, \eta^{2}=.05$, only the U11 group $(d=0.46-0.75)$ differed from the U15 ( $p=.008,0 / 8 / 92$ likely), U17 $(p=.013,0 / 9 / 91$ likely), and $\mathrm{U} 20(p=.045,0 / 5 / 95$ very likely) groups. Although similar to the U13 group $(d=0.26-0.75)$, possibly QCs were identified when comparing the U11 (0/42/58), U15 (0/44/44), and U17 (1/48/52) groups, and likely QCs were identified versus the U20 group $(1 / 15 / 84)$. Finally, the results of the ACUR, $F(5,360)=6.61, p<.001, \eta^{2}=.08$, revealed that the U11 group $(d=1.03)$ was greater than the U15 $(p<.001)$, U17 $(p<.001)$, and U20 $(p=.002)$ groups $(0 / 2 / 98$ very likely for all). In addition, the U13 group $(d=0.52-0.89)$ was also greater than the U17 ( $p=.044$, $0 / 5 / 95$ likely) and U20 ( $p=.036,0 / 8 / 91$ likely) groups. Relevant QCs $(d=0.2-0.67)$ were noted between (a) the U9 group and U11 (94/6/1 likely) and U20 (2/12/86 likely) groups; and (b) the U13 group and U11 (1/50/49 possibly) and U15 (0/24/76 likely) groups.

Table 2. Correlation coefficients between ball velocity and dependent variables according to age.

\begin{tabular}{lrrrrrr}
\hline & U9 & U11 & U13 & U15 & U17 & U20 \\
\hline V $_{\text {foot }}$ & .66 & .83 & .85 & .89 & .89 & .86 \\
$\mathrm{~V}_{\text {ball }} / \mathrm{V}_{\text {foot }}$ & .82 & .72 & .68 & .62 & .51 & .73 \\
LSL & .36 & .57 & .70 & .79 & .70 & .59 \\
$\mathrm{D}_{\text {support-ball }}$ & -.12 & .06 & .40 & .33 & .24 & .12 \\
MRE & -.46 & -.49 & .01 & -.04 & -.19 & -.46 \\
BVE & .07 & -.21 & -.04 & -.12 & -.04 & .23 \\
ACUR & -.24 & -.39 & -.01 & -.08 & -.15 & -.21 \\
\hline
\end{tabular}

Note. Criterion for judgment on correlation coefficients: $r<.2$ (no correlation), $.2 \leq r<.4$ (low correlation), .4 $\leq r<.6$ (moderate correlation), $.6 \leq r<.8$ (moderately high correlation), $r \geq .8$ (high correlation). $\mathrm{V}_{\text {foot }}=$ foot velocity; $V_{\text {ball }} / V_{\text {foot }}=$ ball velocity-to-foot velocity ratio; $\mathrm{LSL}=$ last stride length; $\mathrm{D}_{\text {support-ball }}=$ distance between support foot and ball; $M R E=$ mean radial error; $B V E=$ bivariate variable error; ACUR $=$ accuracy. 
Table 3. Linear and polynomial third-order regressions models between chronological age and dependent variables, including the predictive equations $(n=366)$.

\begin{tabular}{|c|c|c|c|}
\hline Factor & $R^{2}$ & Predictive equation & SEE \\
\hline$V_{\text {ball }}$ & .47 & $V_{\text {ball }}=(5.0743 \times$ Age $)+3.8195$ & 1.77 \\
\hline$V_{\text {foot }}$ & .39 & $V_{\text {foot }}=(3.0105 \times \mathrm{Age})+22.262$ & 1.90 \\
\hline$V_{\text {ball }} / V_{\text {foot }}$ & .28 & $\mathrm{~V}_{\text {ball }} / \mathrm{V}_{\text {foot }}$ ratio $=(0.0264 \times$ Age $)+0.7845$ & 2.05 \\
\hline LSL & .25 & $\mathrm{LSL}=(0.0446 \times \mathrm{Age})+0.7766$ & 2.10 \\
\hline MRE & .07 & MRE $=\left(0.0012 \times\left(\mathrm{Age}^{3}\right)-\left(0.0495 \times\left(\mathrm{Age}^{2}\right)+(0.6143 \times \mathrm{Age})-0.7223\right.\right.$ & 0.53 \\
\hline
\end{tabular}

Correlations between $\mathrm{V}_{\text {ball }}$ and the dependent variables ranged from no correlation to a high correlation. However, the magnitude of these correlations varied across age groups (Table 2). Chronological age also showed moderately high correlations with $\mathrm{V}_{\text {ball }}(r=.68)$ and $\mathrm{V}_{\text {foot }}$ $(r=.67)$, moderate correlations with the $\mathrm{V}_{\text {ball }} / \mathrm{V}_{\text {foot }}$ ratio $(r=.54)$ and LSL $(r=.50)$, low correlations with MRE ( $r=$ -.27) and ACUR $(r=-.26)$, and no correlation with $\mathrm{D}_{\text {support-ball }}(r=.18)$ and BVE $(r=-.18)$. The linear regressions between chronological age and $\mathrm{V}_{\text {ball }}, \mathrm{V}_{\text {foot }}, \mathrm{V}_{\text {ball }} / \mathrm{V}_{\text {foot }}$ ratio, LSL, and a third-order polynomial model with the MRE, including the predictive equation as well as the contribution estimates for the total variance $\left(R^{2}\right)$ and standard error of the estimates, are also described (Table 3). Small (i.e., MRE), medium (i.e., LSL), and large (i.e., $V_{\text {ball }}$, $\mathrm{V}_{\text {foot }}, \mathrm{V}_{\text {ball }} / \mathrm{V}_{\text {foot }}$ ratio) coefficients of determination were found.

\section{Discussion}

The main purpose of the present study was to compare kick performance in young trained soccer players in the U9 to U20 age groups from a large sample, with reference to some biomechanical parameters $\left(V_{\text {ball }}, V_{\text {foot }}\right.$, $\mathrm{V}_{\text {ball }} / \mathrm{V}_{\text {foot }}$ ratio, LSL, $\left.\mathrm{D}_{\text {support-ball }}\right)$ and accuracy measurements (MRE, BVE, ACUR) during soccer kicks. The main findings were that the $\mathrm{V}_{\text {ball }}, \mathrm{V}_{\text {foot}}, \mathrm{LSL}, \mathrm{V}_{\text {ball }} / \mathrm{V}_{\text {foot }}$ ratio, and magnitude of correlation between $\mathrm{V}_{\text {ball }} / \mathrm{V}_{\text {foot }}-$ indicators of foot-ball impact quality-increase linearly with increasing age. Moreover, accuracy parameters appeared to be impaired in adolescence, near to the growth spurt (i.e., PHV age equal to 0 ).

The parameters $\mathrm{V}_{\text {ball }}$ and $\mathrm{V}_{\text {foot }}$ presented a moderately high correlation with age $(r=.68)$. To explain these changes, we rely on the ecological perspective (i.e., dynamic systems approach; Kugler, Kelso, \& Turvey, 1982) applied to motor development that considers the interrelationship between individual, environmental, and task constraints. Firstly, with reference to the constraints of the individual, among the factors that contributed to the results presented herein, one is certainly related to the increasing capacity to produce muscular strength in the lower limbs. Parker, Round, Sacco, and Jones (1990) studied the development of strength through maximal isometric voluntary contractions (MIVC) of the quadriceps in children and adolescents ( $n=267$, aged 5-17 years old) and also identified a trend of a linearly adjusted increase across age groups. In another study, Barber-Westin, Noyes, and Galloway (2006), using an isokinetic dynamometer (ID; $n=224$, aged 9-17 years old), identified that peak torque of knee extension increased progressively from age 9 years (U9 in the present study) to 14 years old (U15 in the present study) and then stabilized when participants were 14 years to 17 years old. We identified a very similar picture for kicking performance (i.e., $\mathrm{V}_{\text {ball }}$ and $\mathrm{V}_{\text {foot }}$ ), with increases from U9 to U15 and signs of stabilization in increases around this age (U15$\mathrm{U} 17 ; p=.22-.29, d=0.28-0.76$, possibly to likely). However, variables from MIVC evaluations (e.g., peak force) and ID (e.g., peak knee extension torque) may reveal only limited kicking information due to differences in task etiology (kick vs. MIVC and ID; McLean \& Tumilty, 1993; Milioni et al., 2016; Vieira et al., 2016). In this way, the development of the muscular system, as previously evaluated, only partially explained kick development. Also, according to a literature review, there would be a positive transference of strength training to kicking performance; however, isolated muscle strength gains could lead to stagnated or possibly impaired kicking skill (Young \& Rath, 2011).

The increased practice time from the younger group to the older groups is a factor that could have contributed to the improvement in velocity (Anderson \& Sidaway, 1994). It is also possible there was an influence of maturation state of the players on kicking performance according to the results of Malina et al. (2007), which revealed that maturity predicted $\left(R^{2}=.21-.29\right)$ a composite skill score (i.e., a sum of performances [Z scores] in ball control, slalom dribbling, passing, and shooting tests). However, when the authors performed the analysis separately, advanced biological maturity status was associated with slightly 
better performance in the shooting test (adjusted $R^{2}=.05-.08$; Malina et al., 2005). Furthermore, our results indicated for the first time that while $\mathrm{V}_{\text {ball }}$ exhibited increases that ranged from $15.2 \%$ to $21.4 \%$ between close groups (except U15 and U17), $\mathrm{V}_{\text {foot }}$ demonstrated smaller increases of $7.7 \%$ to $14.3 \%$ for the same comparisons. Davids, Lees, and Burwitz (2000) argued that attempts at theoretical modeling of the process of coordination and control of kicking in soccer require an understanding of coordination between the movement system and the ball (i.e., constraints of the individual task), rather than only understanding the coordination between the components of the isolated system. In addition, for the first time, our results point out that the magnitude of the correlation between $\mathrm{V}_{\text {ball }}$ and $\mathrm{V}_{\text {foot }}$-assumed as an indirect indicator of impact quality (Apriantono et al., 2006) increased from the U9 group (moderate correlation, $r=.66$ ) to U17 group (high correlation, $r=.89$ ), with a simultaneous increase in another impact quality indicator (i.e., $\mathrm{V}_{\text {ball }} / \mathrm{V}_{\text {foot }}$ ratio; $\mathrm{U} 9,0.99 \pm 0.13$ a.u. $-\mathrm{U} 20$, $1.26 \pm 0.11$ a.u.) and, conversely, a decrease in the magnitude of correlation of the $\mathrm{V}_{\text {ball }} / \mathrm{V}_{\text {foot }}$ ratio and $\mathrm{V}_{\text {ball }}(\mathrm{U} 9, r=.82-\mathrm{U} 17, r=.61$ ). This finding indicates that increasing $\mathrm{V}_{\text {foot }}$ alone does not explain the consequent increases in $\mathrm{V}_{\text {ball }}$ with increasing age. Thus, the factor that contributed to this increase in $V_{\text {ball }}$ was mainly related to the improvement in the quality of the foot-ball interaction at ball impact through the age groups. In other words, older players are more able to transfer higher momentum rates from foot to ball during impact compared with younger players. Interestingly, the kicking performance values of the older group investigated in the present study (U20) were greater compared with age-matched $(63 \pm 7.92-$ $67.68 \pm 5.4 \mathrm{~km} / \mathrm{hr}$; Navarro et al., 2013) and older National Collegiate Athletic Association Division III players (20.2 years old; $81.72 \pm 11.16 \mathrm{~km} / \mathrm{hr}$; Orloff et al., 2008); they were also similar to those of Brazilian elite professional players $\left(\mathrm{V}_{\text {ball }}, 99.74 \pm 8.45\right.$ $\mathrm{km} / \mathrm{hr}$; $\mathrm{V}_{\text {foot }}, 82.31 \pm 7.93 \mathrm{~km} / \mathrm{hr}$; Vieira et al., 2016). Additionally, the U20 group showed greater correlation between $\mathrm{V}_{\text {ball }}$ and $\mathrm{V}_{\text {foot }}$ than reported in a study among adult men (e.g., moderate correlation, $r=.57$; Apriantono et al., 2006), indicating that by this age, players already have a fairly mature pattern of movement to achieve high kicking performance.

A limited number of previous studies have investigated kicking performance in very young U9, U11, and $\mathrm{U} 13$ players and have reported values of $\mathrm{V}_{\text {foot }}$ (U9, 29.24-32.73 km/hr; U11, 33.23-36.56 km/hr; Teixeira \& Teixeira, 2008) that are slightly lower than ours. In the $\mathrm{U} 13$ group, $\mathrm{V}_{\text {ball }}$ was either within the range or moderately lower (58.54-82.06 km/hr; Berjan Bacvarevic et al., 2012; Kapidzic et al., 2014; Wong et al., 2009), and inversely, $V_{\text {foot }}$ was higher than that reported previously ( $48.46 \mathrm{~km} / \mathrm{hr}$; Kapidzic et al., 2014). The majority of the studies in the literature have evaluated players older than U15 (e.g., Apriantono et al., 2006; Juarez et al., 2011; McLean \& Tumilty, 1993; Navarro et al., 2013; Orloff et al., 2008). For the U15 group, our findings were slightly greater than those in a previous study $\left(\mathrm{V}_{\text {ball }}, \sim 60.37-66.26 \mathrm{~km} /\right.$ hr; Berjan Bacvarevic et al., 2012). Regarding the U17 group, our data are either less than $\left(\mathrm{V}_{\text {ball }}, 108.22-\right.$ $109.19 \mathrm{~km} / \mathrm{hr}$; $\mathrm{V}_{\text {foot}}, 87.95-88.92 \mathrm{~km} / \mathrm{hr}$; Juarez et al., 2011) or greater than some references $\left(V_{\text {ball }}, 64 \pm 5\right.$ $79 \pm 6 \mathrm{~km} / \mathrm{hr}$; McLean \& Tumilty, 1993). Changes in coordination patterns between the lower limbs resulting from different angular adjustments in the joints for each age group may be responsible for producing the current results. However, a small number of investigations have been found on this topic with a limited range of age groups (Cerrah et al., 2015; Katis et al., 2015; Teixeira \& Teixeira, 2008), and our data do not allow inferences to be made in this line. Katis et al. (2015) compared two groups of players, one young ( 15 years old) and one adult ( 25 years old) during the instep kick, with only one stride prior to impact. They found that adjustments in the final stages of kicking in the hip (> flexion), knee (> angular velocity), and ankle joints (> plantar flexion, < inversion, and > angular velocity) favored the adults. Nevertheless, the authors themselves reported as a limitation that multistep approaches prior to impact, in addition to increasing performance, may reveal more differences between age groups (Katis et al., 2015). Taking this point into account, we opted in the present study to allow a multistep approach as necessary for each participant before the kicks. Additional research should contribute to the understanding of how the improvement presented here occurred in the quality of foot-ball impact, from childhood to adulthood, as well as the development of proximal-distal transference and kinematic aspects in a variety of age groups.

Unlike velocity, LSL (Kapidzic et al., 2014; Lees et al., 2005) and the $D_{\text {support-ball }}$ (Kapidzic et al., 2014; McLean \& Tumilty, 1993; Orloff et al., 2008) have been little explored to date in young players. In this way, the development of these components was not clear before now. Regarding LSL, only one study presented values of LSL, which were similar to ours (in the U20 group, $1.73 \pm 0.15 \mathrm{~m}$; Lees et al., 2005). Also in this study (Lees et al., 2005), it was reported that LSL influenced $\mathrm{V}_{\text {ball }}$ in the $\mathrm{U} 20$ age group, although with a smaller coefficient ( $r=.42$; see Table 2$)$. In the present 
study, this parameter was identified as correlated, low to moderately high, with the development of $\mathrm{V}_{\text {ball }}$ in all age groups (Table 2), and it also demonstrated increases as a function of age (Table 1). The LSL is important to allow greater range of movement of pelvic retraction, which then provides greater pelvic protraction, in the forward rotation on the kicking side (Lees et al., 2010). Therefore, considering this variable in addition to $\mathrm{V}_{\text {ball }}, \mathrm{V}_{\text {foot}}$, and the $\mathrm{V}_{\text {ball }} / \mathrm{V}_{\text {foot }}$ ratio in battery testing, during the teaching-learning-training process, and in subsequent studies, it is recommended for all age groups from U9 to U20 and to identify if players are suitable for transition to another older group. In contrast, differences in $\mathrm{D}_{\text {support-ball }}$ according to age do not follow the other parameters $\left(\mathrm{V}_{\text {ball }}, \mathrm{V}_{\text {foot}}\right.$, $\mathrm{V}_{\text {ball }} / \mathrm{V}_{\text {foot }}$ ratio, LSL). McLean and Tumilty (1993) and Orloff et al. (2008) reported a $\mathrm{D}_{\text {support-ball }}$ close to ours in U17 $(\sim 0.37 \pm 0.03-0.39 \pm 0.04 \mathrm{~m})$ and $\mathrm{U} 20$ $(0.31 \pm 0.09 \mathrm{~m})$, respectively. In contrast, Kapidzic et al. (2014) found lower values and inverse correlations with the $\mathrm{V}_{\text {ball }}(\mathrm{U} 13-\mathrm{U} 15,0.14 \pm 0.07 \mathrm{~m} ; r=-.45$ ). However, attention is needed in the U13 to U17 groups where an excessively small $\mathrm{D}_{\text {support-ball }}$ could produce slower kicks due to low-to-moderate correlations with $\mathrm{V}_{\text {ball }}$ (Table 2). The absence of correlations between error measurements and $V_{\text {ball }}$ (e.g., no correlation in U13-U17) was in line with the current literature (Navarro et al., 2013; Vieira et al., 2016). Moreover, there was an exception for MRE, which showed moderate inverse correlations in the U9, U11, and U20 groups, indicating that achieving higher $\mathrm{V}_{\text {ball }}$ is not necessarily accompanied by poor ball placement on a target when kicking.

From the calculations of MRE, BVE, and ACUR, we identified that these indicators, which represent measures of error during the kick, follow different dynamics of changes in function of chronological age. These data are not comparable with previous work as they used indirect measures to assess accuracy in young players (Malina et al., 2005, 2007; McLean \& Tumilty, 1993; Rosch et al., 2000; Vaeyens et al., 2006; Vieira et al., 2017; Wilson et al., 2016). Berjan Bacvarevic et al. (2012) pointed out criticism of this type of approach earlier, and we followed their recommendations for the design of the present study. The main results were that MRE, BVE, and ACUR appeared to be impaired in the U11 (MRE, BVE, and ACUR > U15-U20) and U13 (MRE > U17, U20; ACUR > U17 and U20) groups, with a likely extension up to U15 (MRE > U20), and improvements were observed only in the older U17 and U20 groups. During the first pubertal phase (growth spurt; $12-13$ years to $14-15$ years old), there was a marked increase in muscle mass at the same time as muscular strength and, additionally, a rapid increase in height and weight, usually leading to a decrease in coordinative capacity in that control of movements can become compromised. Movements without "precision" are typical at this age (Weineck, 1990). This premise seems also to apply to the ability to kick. Considering that the U9 group did not present the same differences as the older groups, it is possible to suggest that the increased indicators of kicking error suffer from a negative influence of the pubertal period as mentioned earlier (i.e., PHV time), especially in the $\mathrm{U} 13$ group and possibly extending up to the U15 group. Thus, to refine kicking accuracy, we recommend that some specific training sessions focus on instructions such as "hit the center of the target" instead of "kick the ball as fast as possible and try to hit the center of the target" or other instructions focusing mainly on velocities (van den Tillaar \& Ulvik, 2014) often demanded in practical settings.

One of the main limitations of the present study was the choice to perform the kick without the presence of a goalkeeper, which represents a closed motor skill. According to the theoretical base accumulated in recent years (Vieira et al., 2017), the mere presence of a static goalkeeper (Navarro et al., 2013) is a task constraint that actually distracts and affects kicking performance during a penalty. This limitation indicates that caution is required in attempting to transfer the findings to a competitive official match. Furthermore, the U9 and U20 age groups had reduced sample sizes due to the inclusion and exclusion criteria and logistical difficulties (e.g., mandatory necessity of both younger players and their guardians to sign the consent form). In Brazil, due to limited financial resources of several soccer teams to contract new players, U20s are generally also part of professional squads-and we opted not to include them. In addition, future studies with longitudinal approaches are necessary to confirm the occurrence of adaptations in kicking parameters and investigate nondominant limb performance. Up to this point, mechanical factors during kicking have not been assessed during play, mainly due to practical difficulties (Apriantono et al., 2006). Considering that there is still a gap from analysis of the kick to the coaching process (Lees et al., 2010), we tried to provide real contributions to professionals by translating the results into practical terms. To our knowledge, this study was also one of the first on kicking performance to use an integrated analysis approach by applying conventional statistics and magnitude-based inferences, which are contemporary trends (Cohen, 1988; Hopkins et al., 2009). In addition, the current literature has examined a much smaller number of participating 
young soccer players, even in comprehensive work (e.g., $N=106$; Berjan Bacvarevic et al., 2012).

\section{Conclusion}

In summary, responding to the original questions of this study, using 3D and 2D kinematic procedures in a large sample, we concluded that some biomechanical parameters of kicking performance present differentiated development. $\mathrm{V}_{\text {ball }}$ and $\mathrm{V}_{\text {foot }}$ presented increases as a function of the increase in chronological age in a practically linear way, and they were moderately highly correlated with age. The transfer rate in $\mathrm{V}_{\text {ball }}$ to $\mathrm{V}_{\text {foot }}$ and LSL before the kick followed a similar pattern (showing moderate correlations with age). Accuracy parameters presented impairment mainly during the growth spurt period, although there are no (i.e., BVE) to low correlation magnitudes (i.e., MRE and ACUR) with age. These results suggest that different specific training strategies should be applied for each age group.

\section{What does this article add?}

From the results of the present study, we showed that some kicking performance measures $\left(\mathrm{V}_{\text {ball }}, \mathrm{V}_{\text {foot }}, \mathrm{V}_{\text {ball }} / \mathrm{V}_{\text {foot }}\right.$ ratio, and LSL) have moderate to moderately high correlations with individuals' age and present sensitivity to development from childhood (8 years) to the beginning of adulthood (19 years), which is expected to be a very important component during training sessions in youth soccer academies, although from U15 to U17, less pronounced gains are expected. Coaches should consider that during the peak period of growth velocity (growth spurt, U13-U15), accuracy variables are negatively affected, in contrast to the current paradigm (Malina et al., 2005, 2007; Rosch et al., 2000; Vaeyens et al., 2006). Activities that prioritize hitting a target may be beneficial for these age groups. In U13 to U17 age groups, very small $\mathrm{D}_{\text {support-ball }}$ during impact could produce slower kicks. The LSL does not require highly advanced motion capture and measurement systems to be measured and was identified as correlated, low to moderately high, with kicking performance in all groups. Thus, training focused on increasing the range of motion when kicking is also recommended. Coaches should be aware of the reference values and predictive equations (Table 3 ) for the long-term monitoring process to develop the soccer kick. The promotion of young talent to an older age group, a common practice among coaches, could also consider whether players exhibit kicking performance that allows this transition.

\section{Funding}

This work was supported by the Conselho Nacional de Desenvolvimento Científico e Tecnológico [472755/20080,481833/2013-7,487437/2013-6]; Coordenação de Aperfeiçoamento de Pessoal de Nível Superior (CAPES, Brazil).

\section{ORCID}

Luiz H. P. Vieira (D) http://orcid.org/0000-0001-6981-756X

Sérgio A. Cunha (D) http://orcid.org/0000-0003-1927-0142

Renato Moraes (D) http://orcid.org/0000-0001-8029-8334

Fabio A. Barbieri (D) http://orcid.org/0000-0002-3678-8456

Rodrigo Aquino (D) http://orcid.org/0000-0002-4885-7316

Lucas de P. Oliveira (D) http://orcid.org/0000-0002-9347-8286

Martina Navarro (D) http://orcid.org/0000-0001-7822-9647

Bruno L. S. Bedo (D) http://orcid.org/0000-0003-3821-2327

Paulo R. P. Santiago (D) http://orcid.org/0000-0002-9460-8847

\section{References}

Anderson, D. I., \& Sidaway, B. (1994). Coordination changes associated with practice of a soccer kick. Research Quarterly for Exercise and Sport, 65, 93-99. doi:10.1080/ 02701367.1994.10607603

Apriantono, T., Nunome, H., Ikegami, Y., \& Sano, S. (2006). The effect of muscle fatigue on instep kicking kinetics and kinematics in association football. Journal of Sports Sciences, 24, 951-960. doi:10.1080/02640410500386050

Barber-Westin, S. D., Noyes, F. R., \& Galloway, M. (2006). Jump-land characteristics and muscle strength development in young athletes: A gender comparison of 1140 athletes 9 to 17 years of age. American Journal of Sports Medicine, 34, 375-384. doi:10.1177/0363546505281242

Barbieri, F. A., Gobbi, L. T., Santiago, P. R., \& Cunha, S. A. (2015). Dominant-non-dominant asymmetry of kicking a stationary and rolling ball in a futsal context. Journal of Sports Sciences, 33, 1411-1419. doi:10.1080/ 02640414.2014 .990490

Barnett, L. M., van Beurden, E., Morgan, P. J., Brooks, L. O., \& Beard, J. R. (2010). Gender differences in motor skill proficiency from childhood to adolescence: A longitudinal study. Research Quarterly for Exercise and Sport, 81, 162-170. doi:10.1080/02701367.2010.10599663

Berjan Bacvarevic, B., Pazin, N., Bozic, P. R., Mirkov, D., Kukolj, M., \& Jaric, S. (2012). Evaluation of a composite test of kicking performance. Journal of Strength and Conditioning Research, 26, 1945-1952. doi:10.1519/ JSC.0b013e318237e79d

Cerrah, A. O., Şimșek, D., Soylu, A. R., Ertan, H., \& Nunome, H. (2015, June). Developmental differences of kinematic and muscular activation patterns in instep soccer. Paper presented at the 33rd International Conference on Biomechanics in Sports, Poitiers, France.

Cohen, J. (1988). Statistical power analysis for the behavioral sciences. Hillsdale, NJ: Lawrence Erlbaum Associates.

Davids, K., Lees, A., \& Burwitz, L. (2000). Understanding and measuring coordination and control in kicking skills in soccer: Implications for talent identification and skill acquisition. Journal of Sports Sciences, 18, 703-714. doi:10.1080/02640410050120087 
Figueroa, P. J., Leite, N. J., \& Barros, R. M. (2003). A flexible software for tracking of markers used in human motion analysis. Computer Methods and Programs in Biomedicine, 72, 155-165. doi:10.1016/S0169-2607(02)00122-0

Hopkins, W. G., Marshall, S. W., Batterham, A. M., \& Hanin, J. (2009). Progressive statistics for studies in sports medicine and exercise science. Medicine \& Science in Sports \& Exercise, 41, 3-13. doi:10.1249/MSS.0b013e31818cb278

Juarez, D., Lopez de Subijana, C., Mallo, J., \& Navarro, E. (2011). Acute effects of endurance exercise on jumping and kicking performance in top-class young soccer players. European Journal of Sport Science, 11, 191-196. doi:10.1080/17461391.2010.500335

Kapidzic, A., Huremovic, T., \& Biberovic, A. (2014). Kinematic analysis of the instep kick in youth soccer players. Journal of Human Kinetics, 42, 81-90. doi:10.2478/hukin-2014-0063

Katis, A., Kellis, E., \& Lees, A. (2015). Age and gender differences in kinematics of powerful instep kicks in soccer. Sports Biomechanics, 14, 287-299. doi:10.1080/ 14763141.2015.1056221

Kugler, P., Kelso, J. S., \& Turvey, M. (1982). On the control and coordination of naturally developing systems. In J. A. S. Kelso \& J. E. Clark (Eds.), The development of movement control and coordination (pp. 5-78). New York, NY: Wiley.

Lees, A., Asai, T., Andersen, T. B., Nunome, H., \& Sterzing, T. (2010). The biomechanics of kicking in soccer: A review. Journal of Sports Sciences, 28, 805-817. doi:10.1080/02640414.2010.481305

Lees, A., Kershaw, L., \& Moura, F. (2005). The three-dimensional nature of the maximal instep kick in soccer. In T. Reilly \& J. Cabri (Eds.), Science and football V (pp. 65-70). London, England: Routledge.

Malina, R. M., Cumming, S. P., Kontos, A. P., Eisenmann, J. C., Ribeiro, B., \& Aroso, J. (2005). Maturity-associated variation in sport-specific skills of youth soccer players aged 13-15 years. Journal of Sports Sciences, 23, 515-522. doi:10.1080/02640410410001729928

Malina, R. M., Ribeiro, B., Aroso, J., \& Cumming, S. P. (2007). Characteristics of youth soccer players aged 13-15 years classified by skill level. British Journal of Sports Medicine, 41, 290-295. doi:10.1136/ bjsm.2006.031294

McLean, B. D., \& Tumilty, D. M. (1993). Left-right asymmetry in two types of soccer kick. British Journal of Sports Medicine, 27, 260-262. doi:10.1136/bjsm.27.4.260

Milioni, F., Vieira, L. H., Barbieri, R. A., Zagatto, A. M., Nordsborg, N. B., Barbieri, F. A., .. Papoti, M. (2016). Futsal match-related fatigue affects running performance and neuromuscular parameters but not finishing kick speed or accuracy. Frontiers in Physiology, 7, 518. doi:10.3389/fphys.2016.00518

Moore, S. A., McKay, H. A., Macdonald, H., Nettlefold, L., Baxter-Jones, A. D., Cameron, N., \& Brasher, P. M. (2015). Enhancing a somatic maturity prediction model. Medicine \& Science in Sports \& Exercise, 47, 1755-1764. doi:10.1249/ MSS.0000000000000588

Navarro, M., van der Kamp, J., Ranvaud, R., \& Savelsbergh, G. J. (2013). The mere presence of a goalkeeper affects the accuracy of penalty kicks. Journal of Sports Sciences, 31, 921-929. doi:10.1080/02640414.2012.762602
Orloff, H., Sumida, B., Chow, J., Habibi, L., Fujino, A., \& Kramer, B. (2008). Ground reaction forces and kinematics of plant leg position during instep kicking in male and female collegiate soccer players. Sports Biomechanics, 7, 238-247. doi:10.1080/14763140701841704

Parker, D. F., Round, J. M., Sacco, P., \& Jones, D. A. (1990). A cross-sectional survey of upper and lower limb strength in boys and girls during childhood and adolescence. Annals of Human Biology, 17, 199-211. doi:10.1080/ 03014469000000962

Rosch, D., Hodgson, R., Peterson, T. L., Graf-Baumann, T., Junge, A., Chomiak, J., \& Dvorak, J. (2000). Assessment and evaluation of football performance. American Journal of Sports Medicine, 28(Suppl. 5), S29-S39. doi:10.1177/28. suppl_5.s-29

Teixeira, M. C., \& Teixeira, L. A. (2008). Leg preference and interlateral performance asymmetry in soccer player children. Developmental Psychobiology, 50, 799-806. doi:10.1002/dev.20322

Vaeyens, R., Malina, R. M., Janssens, M., Van Renterghem, B., Bourgois, J., Vrijens, J., \& Philippaerts, R. M. (2006). A multidisciplinary selection model for youth soccer: The Ghent Youth Soccer Project. British Journal of Sports Medicine, 40, 928934. doi:10.1136/bjsm.2006.029652

van den Tillaar, R., \& Ulvik, A. (2014). Influence of instruction on velocity and accuracy in soccer kicking of experienced soccer players. Journal of Motor Behavior, 46, $287-$ 291. doi:10.1080/00222895.2014.898609

Vieira, L. H., De Andrade, V. L., Aquino, R. L., Moraes, R., Barbieri, F. A., Cunha, S. A., ... Santiago, P. R. (2017). Construct validity of tests that measure kick performance for young soccer players based on cluster analysis: Exploring the relationship between coaches rating and actual measures. Journal of Sports Medicine and Physical Fitness, 57, 16131622. doi:10.23736/S0022-4707.16.06863-8

Vieira, L. H., de Souza Serenza, F., de Andrade, V. L., de Paula Oliveira, L., Mariano, F. P., Santana, J. E., \& Santiago, P. R. (2016). Kicking performance and muscular strength parameters with dominant and nondominant lower limbs in Brazilian elite professional futsal players. Journal of Applied Biomechanics, 32, 578-585. doi:10.1123/ jab.2016-0125

Weineck, J. (1990). Functional anatomy in sports. Amsterdam, The Netherlands: Elsevier.

Wilson, R. S., James, R. S., Gwendolyn, D., Hermann, E., Morgan, O. J., Niehaus, A. C., \& Smith, M. D. (2016). Multivariate analyses of individual variation in soccer skill as a tool for talent identification and development: Utilising evolutionary theory in sport science. Journal of Sports Sciences, 34, 2074-2086. doi:10.1080/ 02640414.2016.1151544

Wong, P. L., Chamari, K., Dellal, A., \& Wisloff, U. (2009). Relationship between anthropometric and physiological characteristics in youth soccer players. Journal of Strength and Conditioning Research, 23, 1204-1210. doi:10.1519/ JSC.0b013e31819f1e52

Young, W. B., \& Rath, D. A. (2011). Enhancing foot velocity in football kicking: The role of strength training. Journal of Strength and Conditioning Research, 25, 561-566. doi:10.1519/JSC.0b013e3181bf42eb 